\title{
Modeling Vehicle Drivability with Modelica and the Vehicle Dynamics Library
}

\author{
John Griffin ${ }^{1}$ \\ ${ }^{1}$ Modelon AB \\ John Batteh ${ }^{2}$ \\ Ideon Science Park \\ Lund Sweden \\ Johan Andreasson ${ }^{1}$
${ }^{2}$ Modelon, Inc.
Ann Arbor, MI
United States \\ john.griffin@modelon.com john.batteh@modelon.com johan.andreasson@modelon.com
}

\begin{abstract}
This paper highlights the use of multi-domain physical models for simulation of vehicle drivability applications. The models are implemented using the Vehicle Dynamics Library and Engine Dynamics Library from Modelon. The application examples include vehicle launch, vehicle start-stop, and transmission shift events. The examples are structured to illustrate how increasingly sophisticated models provide additional model fidelity or increase the drivability phenomena observed.
\end{abstract}

Keywords: vehicle dynamics; drivability; vehicle modeling; powertrain; engine; transmission; launch; $N V H$

\section{Introduction}

To meet increasingly stringent fuel economy and emissions standards, automotive original equipment manufacturers (OEMs) and suppliers have sought novel technologies to meet customer demand constrained by the regulatory environment. As system complexity increases, the need for increasingly sophisticated analytic tools to perform concept evaluation, capture multi-domain system interactions, and develop and validate control strategies grows. Modelica has been used extensively in the automotive community for modeling and simulation of vehicle dynamics and handling [1], transient engine modeling and performance [2] [3], vehicle thermal management [4], air conditioning systems [5], and vehicle fuel economy and emissions [6].

While customers demand continued refinement in vehicle performance attributes, they also demand no compromises in vehicle comfort and vehicle drivability. Furthermore, many system design or control actions improve one attribute potentially at the expense of another or several others, typically drivability or comfort. Automotive manufacturers are acute- ly aware of the market requirements to achieve best in class levels of vehicle performance and drivability. For example, customers may report drivability related issues such as shift busyness resulting from an increased number of vehicle shifts to optimize fuel economy for transmissions with more gears. Shift performance and feel are also common customer complaints. With increasing use of start-stop technology (see Figure 1), customers experience many more starting events, and their expectations regarding these events differ in driving mode as compared with a single start in park in a garage or parking lot. Vehicle launch with both conventional and especially with start-stop technology can be especially problematic from both a performance and drivability standpoint. With multiple power paths in both conventional and hybrid vehicles, interactions between subsystems can lead to vehicle vibrations typically felt at the seat track by the customer. With the accelerated adoption of dual clutch transmissions, driveline vibrations induced by clutch dynamics are becoming a drivability concern. Variations in clutch friction material, alignment, etc. can affect both nominal performance and drivability, and data to characterize the key components is often not available or considered proprietary by the suppliers.

This paper describes several different vehicle drivability applications. These models are implemented using components from the Modelon Vehicle Dynamics Library (VDL) [1] and Engine Dynamics Library (EDL) [8]. These examples highlight the multi-domain approach needed to simulate vehicle drivability issues. The examples are also structured to illustrate how increasingly sophisticated models provide additional model fidelity or increase the physical phenomena observed. The sample applications in this paper include vehicle launch, vehicle start-stop, and transmission shift events. The applications also include different modeling approaches for the engine with both a conventional automatic and dual clutch transmission. 


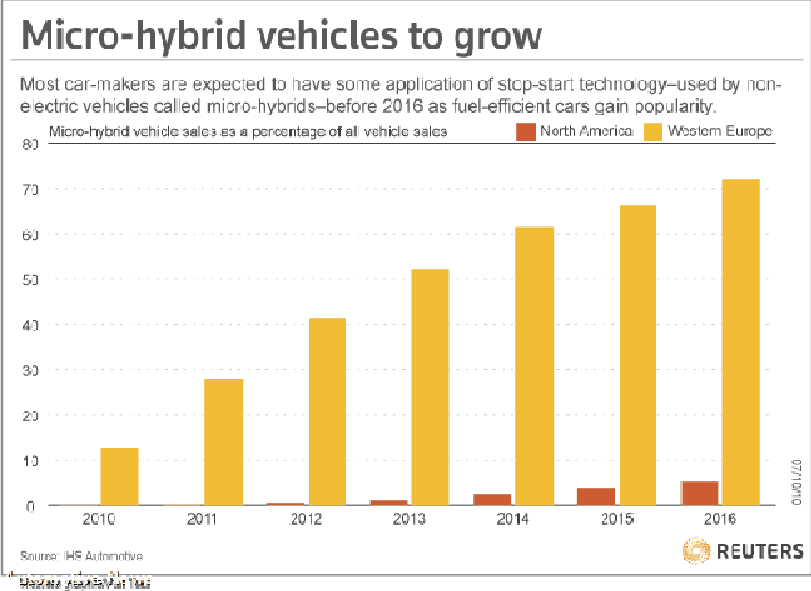

Figure 1. Projections of micro-hybrid vehicles in North America and Europe (reprinted in [7])

\section{Vehicle Modeling}

This section outlines the key multi-domain component and subsystem models that support the subsequent vehicle drivability applications. The main model components are detailed as are the different modeling approaches that can be used to support vehicle drivability applications.

\subsection{Vehicle Model Architecture}

The ability to create configurable model architectures in Modelica is one of the key enablers for architecture-driven development in model-based systems engineering [6]. With core language support for model management and configuration and formal interface definitions, Modelica provides an excellent foundation for distributed, collaborative systems modeling.

The Vehicle Dynamics Library takes an architecture-driven approach to model development and configuration. One of the fundamental guiding principles of VDL is the ability to mix behavioral and physical models to conveniently change between different configurations and also between different levels of detail. From a powertrain perspective, the use of the Rotational3D library [9] is key as it provides a fully-defined representation in $3 \mathrm{D}$ that can easily be reduced to a $1 \mathrm{D}$ representation or vice versa. Within the same architecture, the VDL can support $1 \mathrm{D}$ modeling typically used for conceptual representations early in the product development process to represent the main degrees of freedom to gain early understanding and understand system-level interactions. Furthermore, full 3D representations can be supported which require more extensive parameterization but with a level of detail that provides virtual testing capabilities.
VDL makes use of these Rotational3D connectors to represent the interface cuts between the different components in the powertrain, from the engine to the wheels. Therefore, the architecture has inherent support for plug-and-play compatible exchange between $1 \mathrm{D}$ and 3D components. For example, it is straightforward to combine a 1D driveline in a 3D chassis or a detailed engine model on a lumped chassis.

The component-based interfaces also make it straightforward to switch context, as illustrated for the engine and transmission in Figure 2. In the top model, the engine and transmission are used in a full vehicle template, connected via the driveline to the wheels and the chassis. The other model contains only the engine and the transmission connected to a load, which then can be very simplified, e.g. just a $1 \mathrm{D}$ mass, or also a full chassis with driveline.
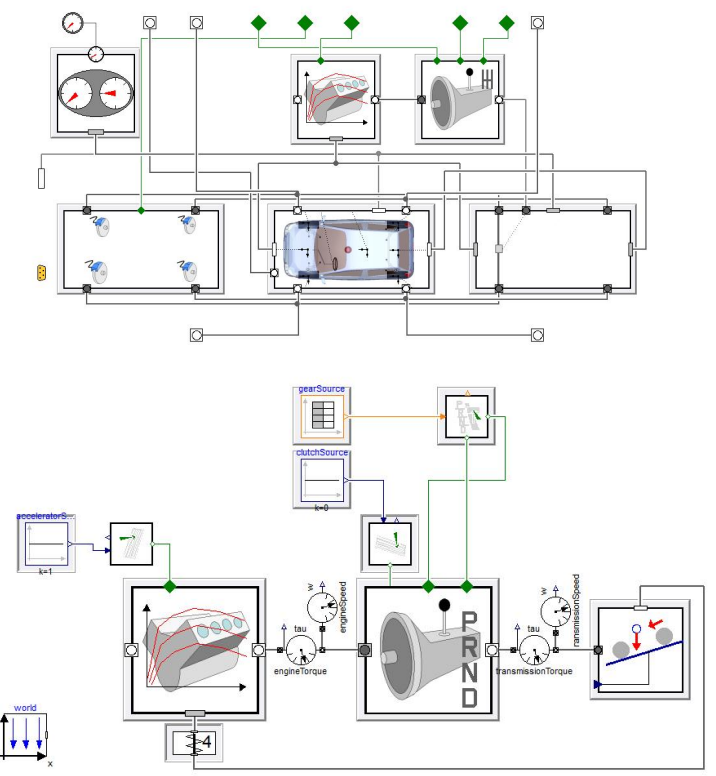

Figure 2. Architectures illustrating the same engine and transmission models in different contexts as shown by the models in a full vehicle representation (top) and together with a load representing the vehicle (bottom)

\subsection{Engine}

Modelica has been used extensively for simulating detailed engine transient response including combustion [2] [3]. Proper representation of the engine dynamic response is critical for vehicle drivability efforts. Both the mean and fluctuating component of the engine torque production can induce undesirable vehicle driveline response. The various delays in the engine due to controls scheduling constraints, actuator response, air path dynamics, and fueling dynamics can also be important. Engine inertial response (piston mass, crank/slider inertia, crankshaft inertia) can also be an important consideration in dynamic 
simulation, especially for cranking, launch, and startstop events.

Typical engine modeling approaches for drivability applications include the following:

- Mean value modeling based on maps and actuator inputs

- Mean value modeling including air path (intake and exhaust) dynamics

- Mean value modeling with superimposed torque fluctuations at the crankshaft

- High frequency modeling with multiple cylinders based on input cylinder pressure

- High frequency engine modeling with multiple cylinders and physics-based combustion modeling

These approaches cover a range of predictive capability, computational effort, and input/calibration data requirements. Furthermore, the level of expertise required to implement and validate the various models varies greatly.

The engine components in the Vehicle Dynamics Library support multiple approaches for modeling the engine and crankshaft. These approaches range from a pure map-based mean value approach to a high frequency approach based on cylinder pressures. Figure 3 shows an I4 engine with a cylinderbased pressure calculation and a distributed model of the engine bottom. An analytic representation can be used for the pressure calculation to allow faster simulation with a standard table for torque as a function of throttle and engine speed required for parameterization. This model calculates a pressure trace profile using spark timing with adjustable shape parameters and induces the appropriate amount of crankshaft torque fluctuation. A sample cylinder force and engine animation for the I4 engine is shown in Figure 4. A full tabular representation for the cylinder pressure as a function of crankangle, throttle, engine control settings, and engine speed can also be used.

To support additional modeling options for the engine, engine models from the Engine Dynamics Library [8] can be integrated into the VDL vehicle architecture. The Engine Dynamics Library currently provides mean value engine modeling capability including the air path dynamic effects, exhaust modeling, and thermal effects. The focus of the library is real-time like simulation of gas exchange and mean value torque production to support engine optimization and evaluation of engine control strategies. A turbocharged, spark-ignited engine model using EDL is detailed in Section 3 and integrated within the VDL architecture for use in a start-stop application.
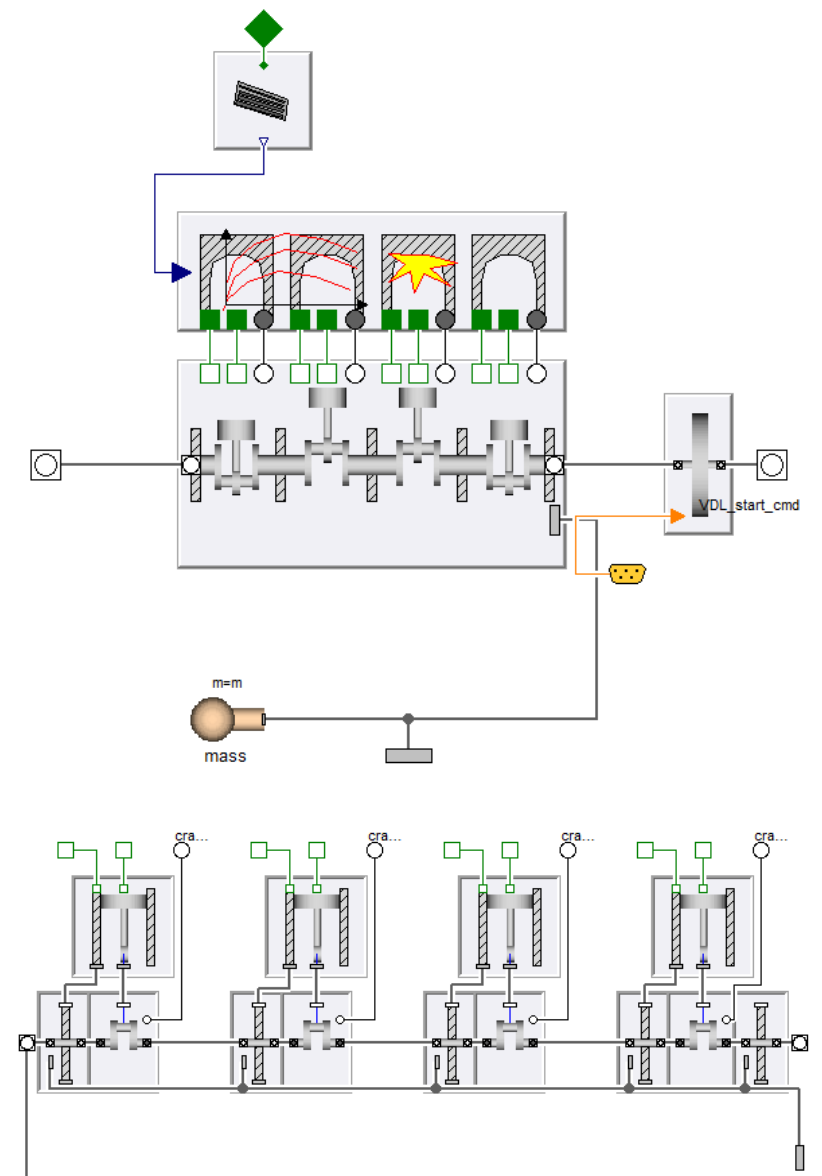

Figure 3. I4 engine with cylinder pressure calculation and dynamic bottom (top) and detail for engine block showing piston and crankshaft models (bottom)

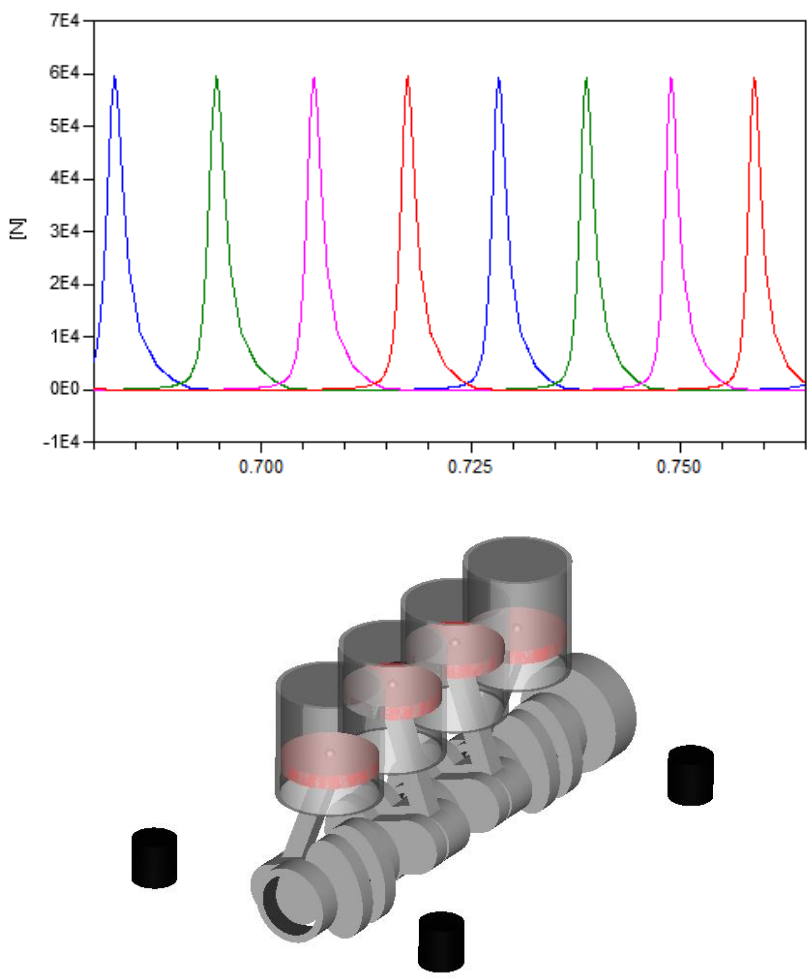

Figure 4. Cylinder force (top) and engine animation (bottom) for I4 engine 


\subsection{Transmission and Driveline}

Several transmission implementations are available in the Vehicle Dynamics Library, including representations of automatic, manual, and CVT transmissions. Within the architecture of Vehicle Dynamics Library, it is certainly possible to implement custom transmission models including both $1 \mathrm{D}$ and $3 \mathrm{D}$ effects, backlash, friction, etc.

Dual clutch transmissions (DCT) are becoming increasingly popular due to the projected fuel economy benefits resulting from the removal of the torque converter, dry clutch technology, etc. However, many of these changes also can pose new performance (no torque multiplication from torque converter), drivability (no damping from torque converter due to fluid coupling), and control (managing clutch to clutch transitions for gear changes) challenges. Thus, dual clutch transmissions are often mated with a dual mass flywheel to provide the required damping but with increased inertia.

To illustrate some of these challenges in the following drivability applications, the simple dual clutch gearbox model shown in Figure 5 was implemented and integrated into a transmission model. Since this model is primarily for demonstration purposes, the control interfaces are simplified. This model implementation should also be considered as functional as no detailed parameterization data (clutch, gearing, etc.) was available to support a more detailed model implementation for the purposes of this paper. For a full treatment of a dual clutch transmission and associated control, the interested reader is referred to [0].

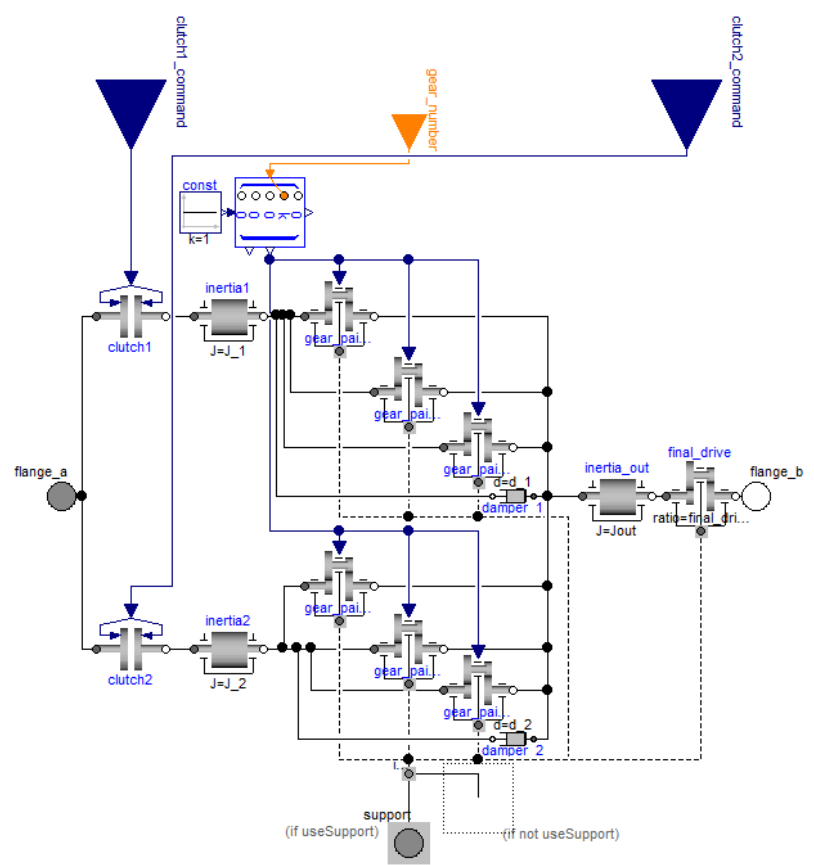

Figure 5. Dual clutch transmission gearbox
The Vehicle Dynamics Library provides both components and assembled subsystems to model various driveline implementations (front, rear, allwheel drive). Components are available for gears, gear pairs, clutches, shafts, differentials, etc. The shaft models are implemented such that geometric effects such as joint effects and bend angles can be modeled if needed. Pure 1D rotational components can be used as well with the 1D/3D structure provided by the library architecture. Figure 6 shows a rear wheel drive driveline model with a transmission shaft, rear differential, and geometric half shafts; this model is used in the vehicle model examples shown in Section 3.

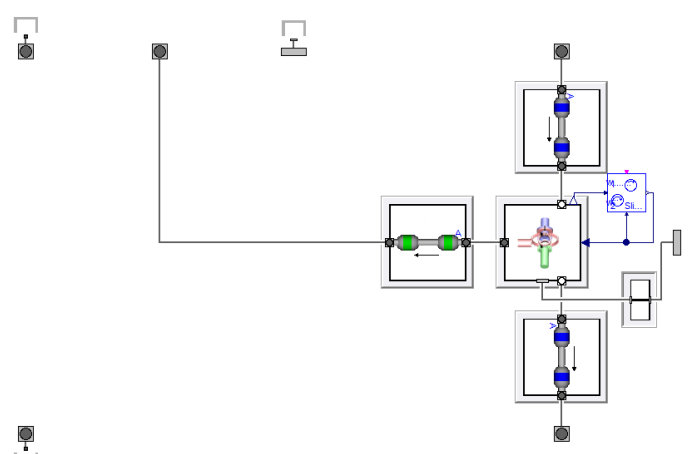

Figure 6. Rear wheel drive driveline

\subsection{Chassis}

The Vehicle Dynamics Library includes a wide range of suspension models with fidelity levels that span from planar models to fully geometric, elastokinematic models. Lower fidelity suspension models are more desirable in drivability simulations for many reasons. First, the engineers performing drivability simulations are mainly interested in straightline behavior and longitudinal dynamics. For these types of simulations, unlike in handling simulations, it is not important to accurately represent how the wheel moves with respect to the chassis for a given wheel travel and load condition. A key benefit of using lower fidelity suspension models is that information required to represent them is significantly lower than a more complex physical model.

Historically, lower fidelity models have included the following representations:

- Planar suspension

- Equivalent roll stiffness

- Lumped mass

- Swing arm

Each of these suspension models are available to be used within VDL. The lower fidelity suspension models have the same interface as the more complex physical suspension models. This modular approach 
ensures that either representation can used in the chassis at any time.

The range of chassis implementations are described in more detail below and shown in Figure 7 via animations from VDL. The example applications in Section 3 include simulations within this range of chassis models.

Pitch model: The suspension is modeled as a rigid axle that can only translate vertically with respect to the chassis body. Ride stiffness is modeled using vertical springs and dampers. This model allows the chassis to heave and pitch.

Equivalent roll stiffness model: The suspension is modeled as a rigid axle that rotates about a single axis. Roll stiffness is modeled using a torsional spring and damper. This model allows the chassis to roll.

Lumped mass model: The suspension is modeled to allow each wheel to translate vertically with respect to the chassis body. Ride and roll stiffness is modeled using vertical springs and dampers. This model allows the chassis to heave, pitch and roll.

Swing Arm model: The suspension is modeled to allow each wheel to swing on a control arm about a single axis. Ride and roll stiffness is modeled using vertical springs and dampers. This model allows the chassis to heave, pitch and roll.

Tabular model: The suspension is modeled using tables that define the kinematic and compliant model of the wheel with respect to the chassis body. The model allows the chassis to heave, pitch and roll.

In order to use these models to represent a chassis for drivability work, it is only necessary to provide the chassis mass, track width, wheelbase and approximate spring rates. Since many engineers who work on powertrain response and drivability applications do not have ready access to the geometry required for more detailed chassis models, a small set of parameterization data can result in significant reductions in model development time.

While lower fidelity dynamic models typically are sufficient for vehicle drivability work, there are some applications which might require more detailed chassis representations. For example, some launch maneuvers might require more detailed models to observe anti-squat or differential/axle windup effects. With the architecture and associated components from the Vehicle Dynamics Library, the various chassis representations are plug-in compatible such that full multibody representation can be seamlessly integrated.

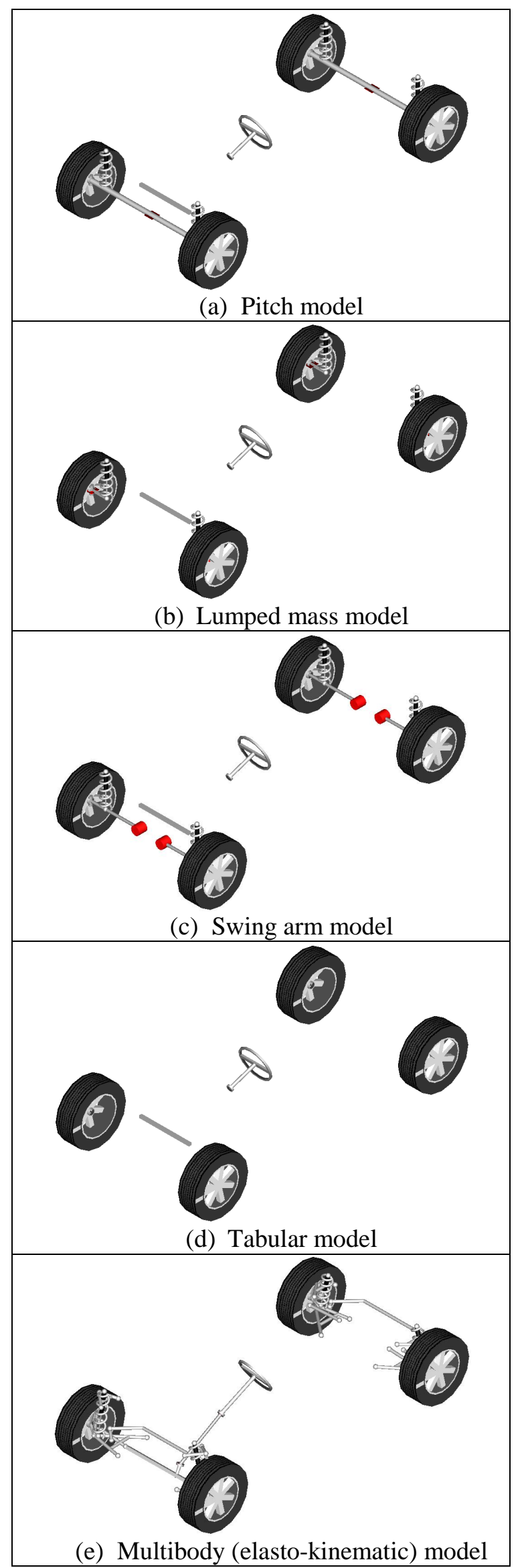

Figure 7. Range of chassis implementations from VDL 


\subsection{Powertrain Mounts}

Powertrain mounts are another critical element for modeling of vehicle drivability events. The movement of the powertrain on the mounts affects the torque transfer in the driveline as well as providing a transfer path for vibrations to the vehicle seat track. Mount design affects vehicle performance and comfort and requires a simulation environment capable of transient simulations over critical maneuvers of interest (idle, launch, tip in- tip out, etc.).

The VDL architecture includes a configurable component for modeling the powertrain mounts. The inherent 3D support makes addition of reaction forces and torques straightforward to capture the true dynamics of the system. The component can be configured for the number of mounts, mount locations, and also the characteristics of the mount behavior. For example, Figure 8 shows bushing compression (gray areas) due to rotation of the differential housing when the torque is transmitted from the longitudinal to lateral direction. These effects require a 3D representation of the driveline and differential which are readily implemented using components from the Vehicle Dynamics Library.

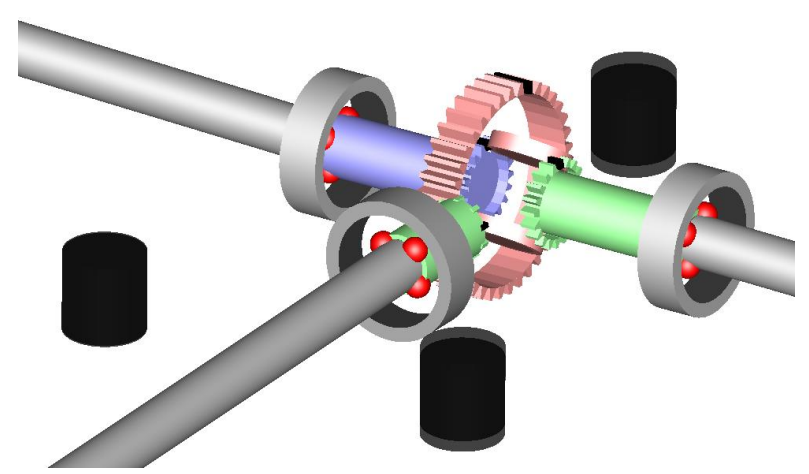

Figure 8. Differential housing wind-up

\section{Application Examples}

Using the component models outlined in the previous section, this section provides several example models illustrating the impact of modeling approaches on vehicle drivability response.

\subsection{Vehicle Launch}

Figure 9 shows a model configured for wide open throttle (WOT) vehicle launch from idle with an automatic transmission. This implementation includes a simple mapped engine. Figure 10 shows some sample results from the launch and subsequent shift events with the tabular chassis model. Note the acceleration disturbances around each shift event. The- se sorts of disturbances can be mitigated by appropriate torque control and shift coordination between the engine and transmission. The development and optimization of such coordinated control is readily achieved using model-based systems engineering approaches with VDL.

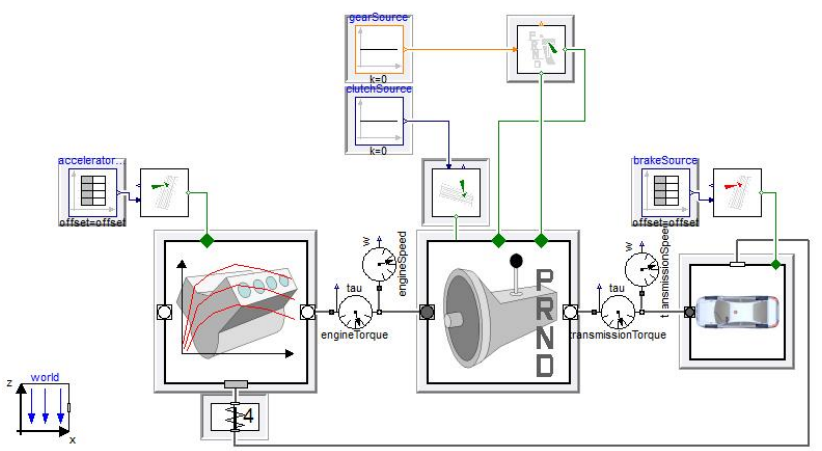

Figure 9. Vehicle launch model with automatic transmission
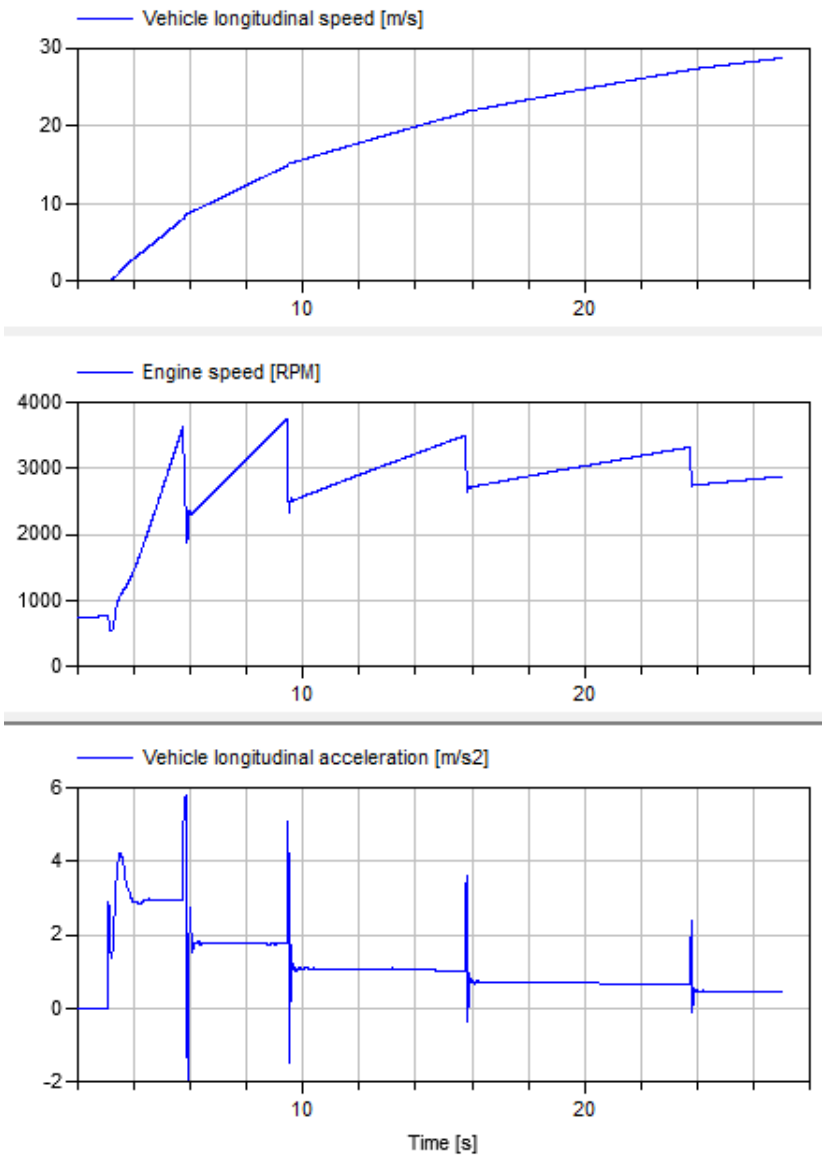

Figure 10. Vehicle launch from idle and shift results with automatic transmission

Figure 11 shows comparisons between the chassis pitch and roll angle for the various chassis model implementations. For the chassis pitch angle, the pitch, lumped mass, and swing arm models provide similar results as do the tabular and multibody mod- 
els. For the chassis roll angle, the pitch model shows essentially no roll while the results from the lumped mass and swing arm models are grouped together as are the results from the tabular and multibody models.

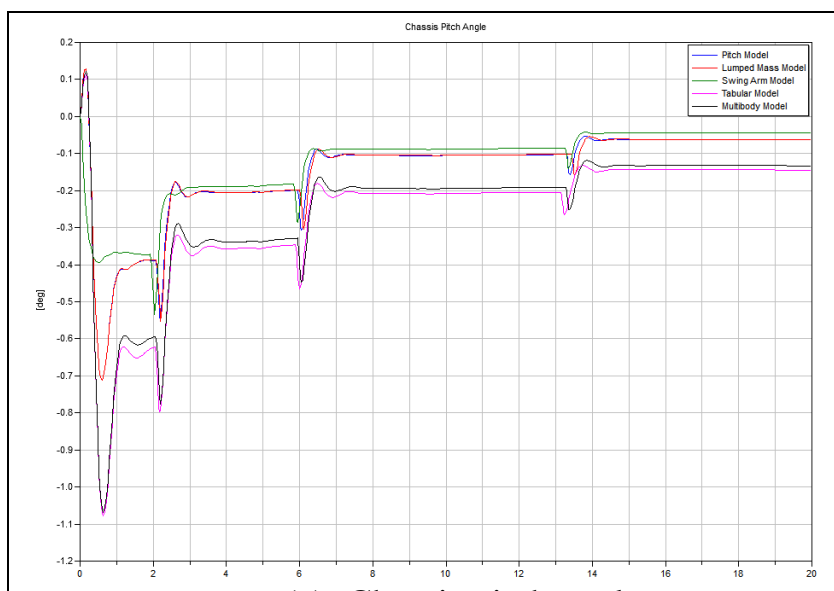

(a) Chassis pitch angle

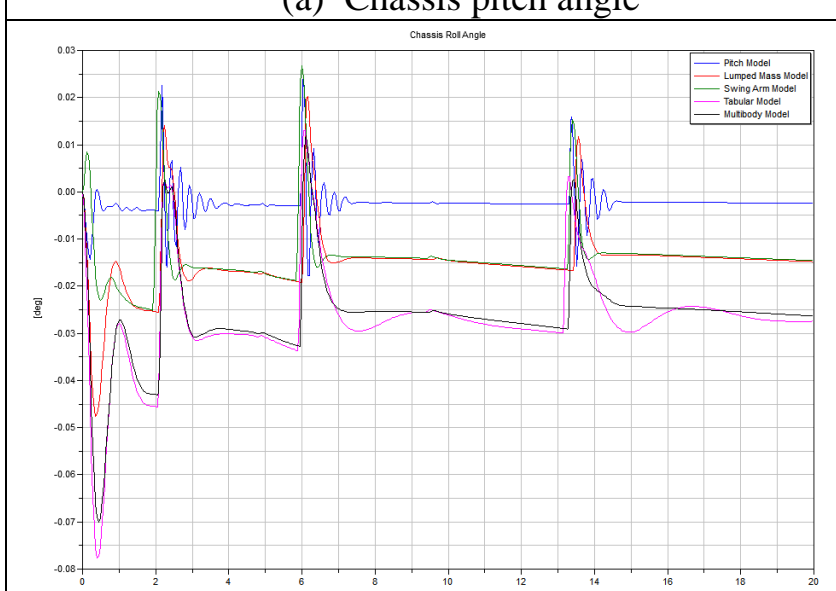

(b) Chassis roll angle

Figure 11. Chassis pitch (a) and roll angle (b) for the various chassis model implementations

Figure 12 shows a model configured for wide open throttle vehicle launch with a dual clutch transmission. Note that the only changes from Figure 9 are the transmission swap and associated transmission control specification (open loop in this example). Figure 13 shows some sample results from the initial launch and first 1-2 shift event. Note the driveline disturbances shown during the shift due to a poorly executed clutch to clutch transition. With a dual clutch transmission, the key to shift feel and performance is managing this transition thus highlighting the importance of clutch modeling (frictional characteristics, actuation, dynamic response, etc.) and controls for this type of transmission. Given the complex dynamic response, a model-based systems engineering approach is required for multi-attribute balancing of performance and drivability.

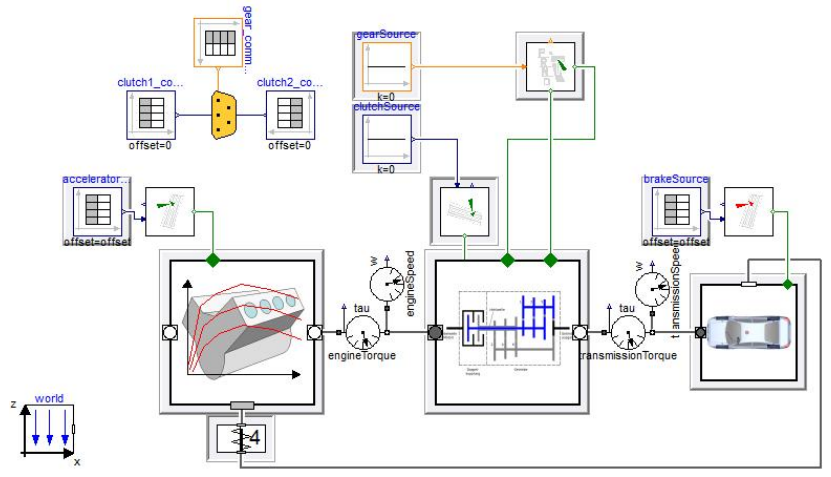

Figure 12. Vehicle launch model with dual clutch transmission
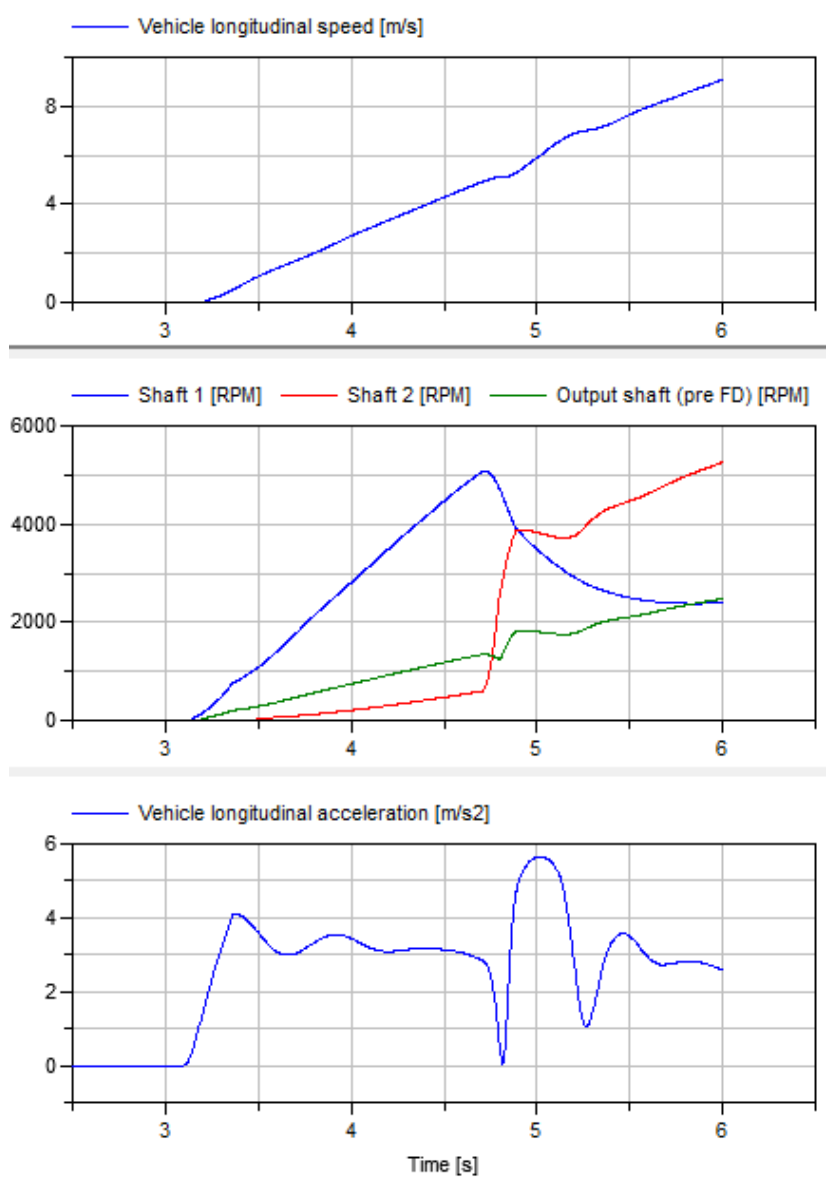

Figure 13. Initial launch and 1-2 shift results for dual clutch transmission

The vehicle launch models illustrate the power of a modeling architecture and supporting component models such that the focus of the model can easily be shifted from full vehicle to powertrain with different levels of complexity for the chassis and driveline. This approach allows model detail to be implemented in the areas where it is critical for observing the dynamic phenomena of interest while allowing model simplifications in other areas. This approach enables a balance between model complexity, computa- 
tional effort, and also parameterization effort given that more detailed models typically require more detailed data for parameterization.

\subsection{Start-Stop}

Start-stop technology on mild/micro-hybrid vehicles offers compelling fuel economy benefits with elimination of idle fuel consumption. While the fuel economy benefits are clear, the drivability impact can be significant if the restarts are not managed well. Customers experience many more starting events, and their expectations regarding these events differ when driving as compared with a single start in park in a garage or parking lot. The engine must be quickly cranked from rest and able to meet driver demand for the subsequent launch event. The engine cranking, fueling, airflow, and transmission engagement events must be managed to provide quick restart performance while minimizing driver disturbances felt at the seat track.

As with all drivability applications, the appropriate choice of model for the engine, transmission, driveline, and chassis depends on the overall goal of the simulation and frequency range of interest. Potential applications include the following:

- Starter motor, battery, and electrical system sizing

- Model-based controls development

- Design of driveline isolation components (damper, dual mass flywheel, etc.)

- Powertrain mount design

- Launch performance and sensitivity to physical and controls parameters

- Driveline response over the range of engine speeds and torques seen during crank, initial combustion, and run-up phases

For start-stop applications, one of the key modeling choices involves the engine dynamic response and resulting torque signature. While mean value modeling approaches may be sufficient for some applications, others may require that the torque pulses at the crankshaft are represented as they may be key for the drivability phenomena of interest. The start-stop examples that follow cover a range of engine modeling approaches from the Vehicle Dynamics and Engine Dynamics Libraries.

As described in Section 2.2, VDL includes engine models capable of producing fluctuating torque at the crankshaft. Using the model shown in Figure 3 with a separate cylinder head and distributed engine bottom, a map-based mean torque can be analytically transformed into a pressure calculation including the influence of control parameters on the pressure shape. This representation, while approximate when compared to a detailed engine cycle simulation, does not require detailed engine characterization for model development (i.e. intake and exhaust system flow characteristics, valve profiles and discharge coefficients, combustion characteristics, etc.) and can provide the appropriate amount of crankshaft torque fluctuation in a computationally efficient manner. It should be noted that appropriate care must be taken when calculating the mean torque to account for the various delays in the engine response which are not physically modeled (i.e. throttle and airflow response, fueling response, manifold filling and emptying, etc.).

Within the architecture provided by VDL, a plugcompatible I4 engine model with fluctuating torque is chosen for the replaceable engine subsystem shown in Figure 9. Results from start-stop simulations with this engine with an automatic transmission are shown in Figure 14. These results show the impact of the starter on initial launch behavior. When the starter disengages early, the engine speed drops after the initial crank until the runup phase begins due to the initial firing events. With normal starter disengagement, the engine speed smoothly increases during crank followed by the initial combustion events. If the starter torque is also increased, the expected increase in engine speed is observed. Note the similar trends in the vehicle speed response.
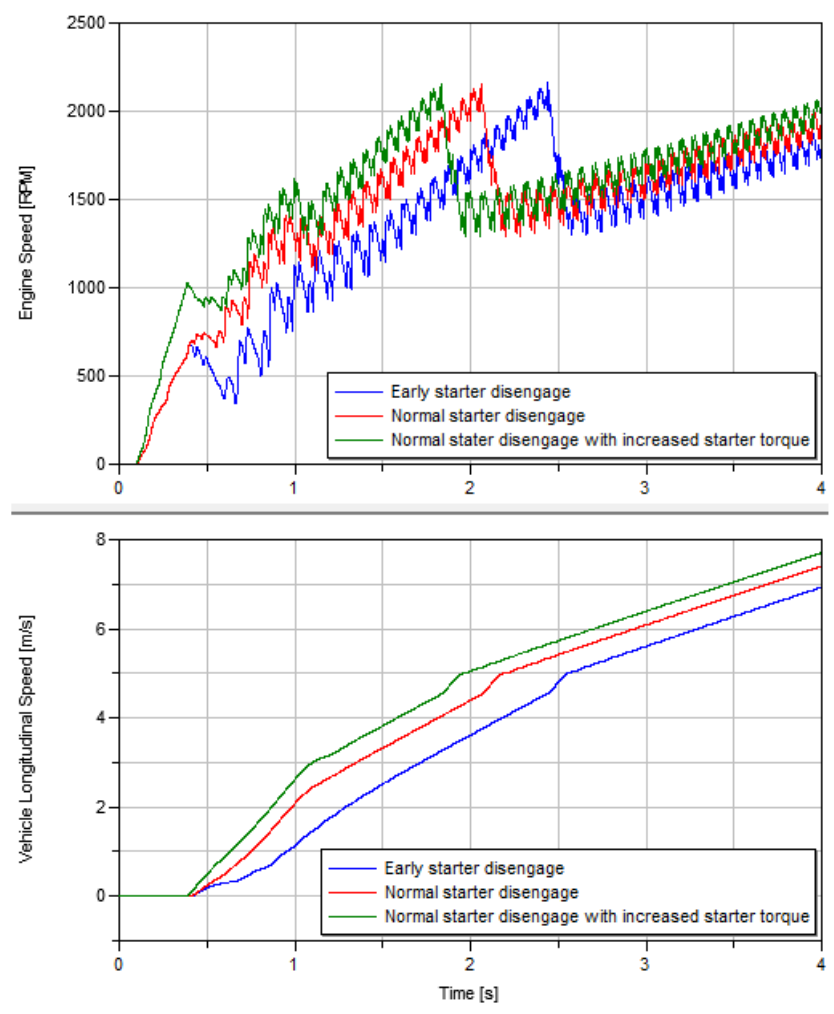

Figure 14. Start-stop response with automatic transmission [engine speed (top) and vehicle speed (bottom)] 
To support additional modeling options for the engine, engine models from the Engine Dynamics Library [8] can be integrated into the VDL vehicle architecture. The Engine Dynamics Library currently provides mean value engine modeling capability including the air path dynamic effects, exhaust modeling, and thermal effects. With the physical modeling approach in the EDL, the engine model responds naturally to changes in actuation and control commands based on the individual component characteristics. Appropriate delays in engine response are also simulated via the physical characteristics of the components. The component-based approach in EDL also allows advanced concept evaluation, component sizing and optimization studies, and modelbased controls development.

Figure 15 shows a turbocharged, spark-ignited engine model using EDL. The model includes lumped representations of the intake air path (light blue connections), exhaust air path with EGR loop (orange connections), simplified cooling path (blue connections), key heat transfer effects in the charge air cooler, cylinder, and exhaust manifold, and a turbocharger with wastegate. The model is roughly parameterized for a $2 \mathrm{~L}$ engine. For the purposes of this paper, a battery and electric motor are added to the turbo system to provide "eboost" capability. This engine model is then integrated into the VDL architecture with the dual clutch transmission shown in Figure 12.

Figure 16 shows results from the start-stop launch with the EDL engine model, dual clutch transmission, and tabular chassis model. The results are for an aggressive launch with varying levels of eboost motor assist applied in the first second of the launch event. The simulations show the initial cranking event, engine run-up due to combustion, and 1-2 shift with the dual clutch transmission which occurs around $1.5 \mathrm{~s}$. With increasing motor assist, the turbo speed increases much more rapidly than would be possible with the compressor driven by the turbine alone, especially since the early combustion events do not provide significant exhaust enthalpy to drive the turbine (i.e. lower mass flow rates and lower exhaust temperatures). The increased turbo speed results in additional boost and thus additional engine torque as shown in Figure 17 and higher engine speeds. With a dual clutch transmission, the coupling between engine and transmission can be managed to optimize the overall launch event by controlling the timing of wheel torque subject to drivability constraints.

While the EDL currently provides a mean value modeling approach for the engine, it is also possible to generate a fluctuating torque using the same mod- els shown in Figure 3 by replacing the tabular torque map from VDL with the dynamic EDL model. With this approach, the engine model remains physicsbased but can also provide fluctuating crankshaft torque for drivability applications without significant additional computational expense.

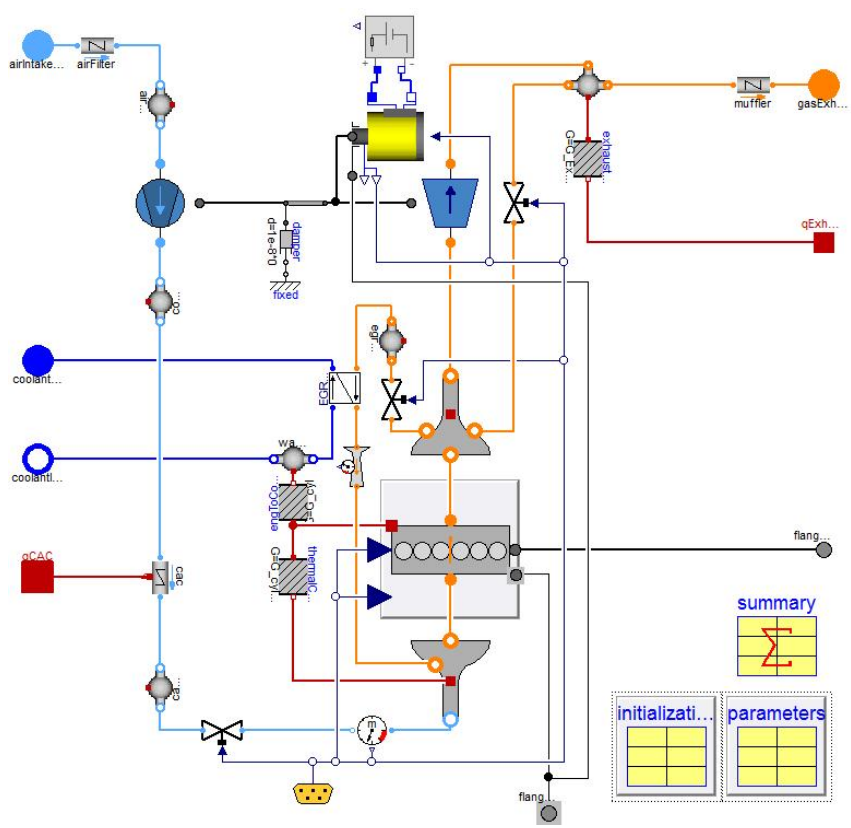

Figure 15. Turbocharged, spark-ignited engine model using the Engine Dynamics Library
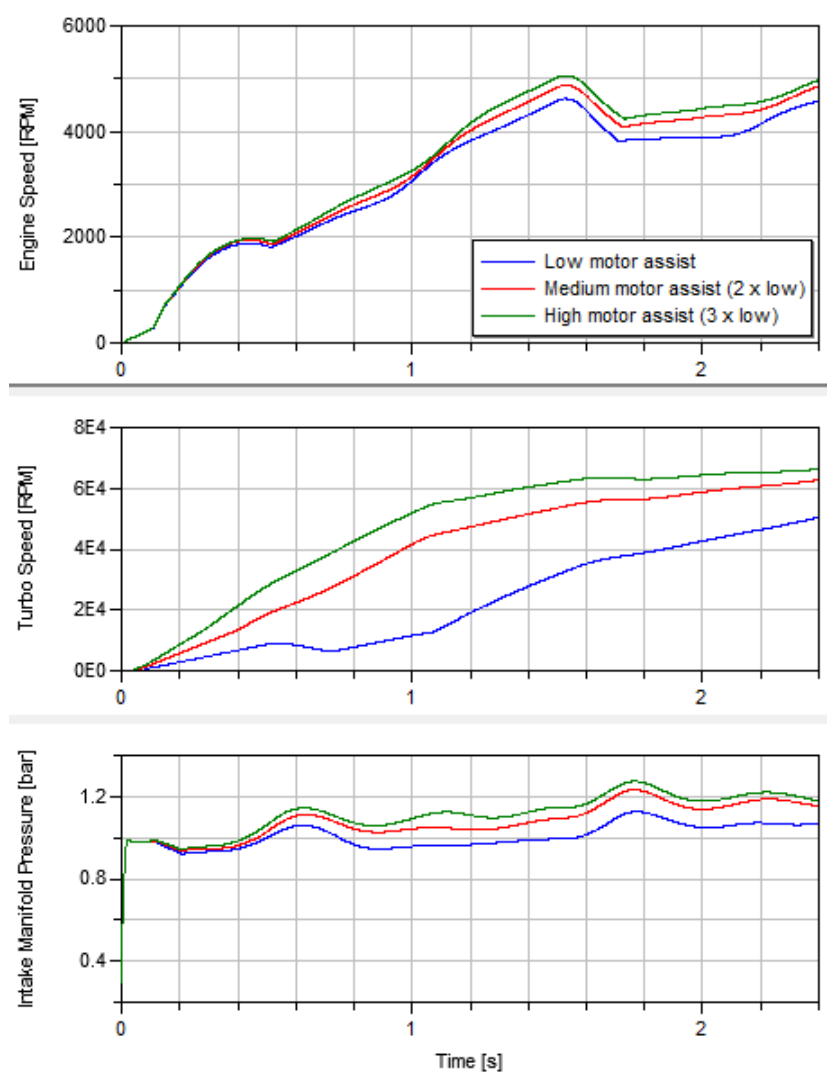

Figure 16. Start-stop response with Engine Dynamics Library engine model and dual clutch transmission 


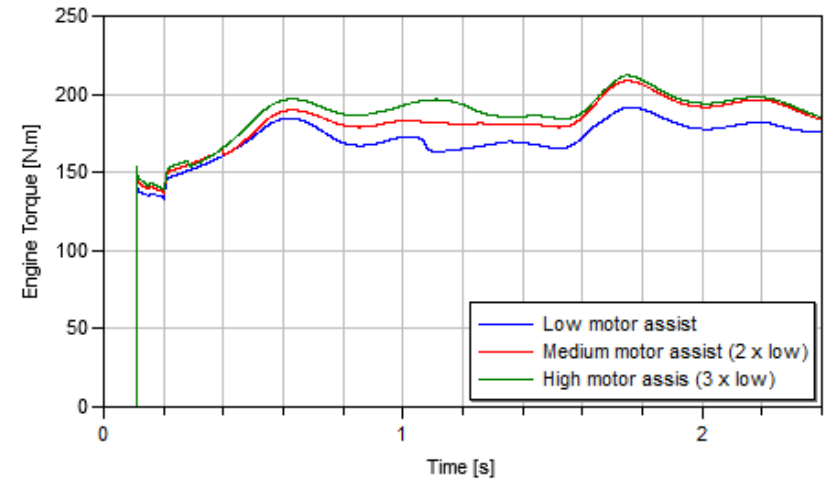

Figure 17. Engine torque for start-stop launch

\section{Conclusions}

Several application examples focused on vehicle drivability have been detailed. These application examples include vehicle launch, start-stop, and transmission shift performance. These examples highlight the use of sophisticated model libraries with different levels of fidelity for key components such as the chassis along with different modeling approaches for the engine and both automatic and dual clutch transmissions. The model libraries illustrate the multi-domain approach required to simulate vehicle drivability. Using the flexible model architecture from the Vehicle Dynamics Library, the various examples are seamlessly configured using plugcompatible variants. The examples are structured to illustrate how increasingly sophisticated models provide additional model fidelity or increase the drivability phenomena observed. An engine model created using the Engine Dynamics Library is coupled with the models and architecture from the Vehicle Dynamics Library to illustrate a range of engine modeling approaches to support vehicle drivability applications.

\section{References}

[1] Andreasson, J., "The Vehicle Dynamics Library: New Concepts and New Fields of Application", Proceedings of $8^{\text {th }}$ International Modelica Conference, 2011.

[2] Newman, C., Batteh, J., and Tiller, M., "Spark-Ignited-Engine Cycle Simulation in Modelica", Proceedings of $2^{\text {nd }}$ International Modelica Conference, pp. 133-142, 2002.

[3] Batteh, J. and Newman, C., "Detailed Simulation of Turbocharged Engines in Modelica", Proceedings of $6^{\text {th }}$ International Modelica Conference, pp. 69-75, 2008.
[4] Wang, et al., "Integrated Thermal Management Simulations: Evaluating the Effect of Underhood Recirculating Airflows on ACSystem Performance, Proceedings of $7^{\text {th }}$ International Modelica Conference, pp. 413422, 2009.

[5] Eborn, et al., "AirConditioning - a Modelica Library for Dynamic Simulation of AC Systems, Proceedings of $4^{\text {th }}$ International Modelica Conference, pp. 185-192, 2005.

[6] Batteh, J. and Tiller, M., "Implementation of an Extended Vehicle Model Architecture in Modelica for Hybrid Vehicle Modeling: Development and Applications", Proceedings of $7^{\text {th }}$ International Modelica Conference, pp. 823-832, 2009.

[7] Niedermeyer, E., "The Shocking Truth About Start-Stop Systems", http://www.thetruthaboutcars.com/2011/06/t he-shocking-truth-about-start-stop-systems/, June 29, 2011.

[8] Andersson, D., and Dahl, J., 2012, "Gas Exchange and Exhaust Condition Modeling of a Diesel Engine using the Engine Dynamics Library", Proceedings of $9^{\text {th }}$ International Modelica Conference, 2012.

[9] Andreasson, J. and Gäfvert, M., "Rotational3D-Efficient modelling of 3D effects in rotational mechanics", Proceedings of $6^{\text {th }}$ International Modelica Conference, pp. 515520, 2008.

[10] Isernhagen, H. and Guhmann, C., "Modelling of a Double Clutch Transmission with an Appropriate Controller for the Simulation of Shifting Processes", Proceedings of $6^{\text {th }}$ International Modelica Conference, pp. 333-339, 2008. 